\title{
Metabolic Regulation of Macrophage Activation
}

\author{
Ourania Kolliniati ${ }^{a, b}, c$ Eleftheria leronymaki ${ }^{a, c}$ Eleni Vergadi ${ }^{b}$ \\ Christos Tsatsanis ${ }^{a, c}$

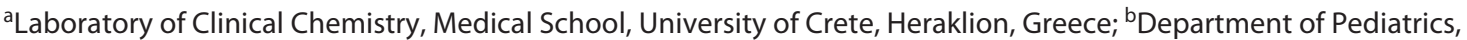 \\ Medical School, University of Crete, Heraklion, Greece; 'Institute of Molecular Biology and Biotechnology, FORTH, \\ Heraklion, Greece
}

\section{Keywords}

Metabolism · Inflammation · Insulin · Cancer · Macrophage · Obesity · Diabetes

\begin{abstract}
Macrophages, the central mediators of innate immune responses, being in the first-line of defense, they have to readily respond to pathogenic or tissue damage signals to initiate the inflammatory cascade. Such rapid responses require energy to support orchestrated production of pro-inflammatory mediators and activation of phagocytosis. Being a cell type that is present in diverse environments and conditions, macrophages have to adapt to different nutritional resources. Thus, macrophages have developed plasticity and are capable of utilizing energy at both normoxic and hypoxic conditions and in the presence of varying concentrations of glucose or other nutrients. Such adaptation is reflected on changes in signaling pathways that modulate responses, accounting for the different activation phenotypes observed. Macrophage metabolism has been tightly associated with distinct activation phenotypes within the range of M1-like and M2-like types. In the context of diseases, systemic changes also affect macrophage metabolism, as in diabetes
\end{abstract}

karger@karger.com www.karger.com/jin

Karger"

BOPEN ACCESS
(C) 2021 The Author(s)

Published by S. Karger AG, Basel

This is an Open Access article licensed under the Creative Common Attribution-NonCommercial-4.0 International License (CC BY-NC) (http://www.karger.com/Services/OpenAccessLicense), applicable to the online version of the article only. Usage and distribution for commercial purposes requires written permission. and insulin resistance, which results in altered metabolism and distinct activation phenotypes in the adipose tissue or in the periphery. In the context of solid tumors, tumor-associated macrophages adapt in the hypoxic environment, which results in metabolic changes that are reflected on an activation phenotype that supports tumor growth. Coordination of environmental and pathogenic signals determines macrophage metabolism, which in turn shapes the type and magnitude of the response. Therefore, modulating macrophage metabolism provides a potential therapeutic approach for inflammatory diseases and cancer.

(c) 2021 The Author(s)

Published by S. Karger AG, Basel

\section{Introduction}

Macrophages are present in all tissues and devoted to maintain tissue integrity, homeostasis, and balance. They constitute a cell population of diverse origin since they arise from distinct cell lineages during embryonic development [1]. Tissue-specific resident macrophages differentiate from precursor stem cells of the tissue, whereas blood circulating monocytes originate from common myeloid progenitor cells in the bone marrow [2]. 
Macrophage adaptation and heterogeneity are reflected on gene expression patterns and cytokine production, orchestrated in order to finely tune inflammatory responses. Ranging from recognition of opportunistic agents and clearance of apoptotic cells to antigen presentation and stimulation of the adaptive immune system, macrophages exhibit rapid adaptation and acute response in several conditions [3]. In order to mount such responses, macrophages are equipped with a wide range of cell surface receptors that are able to recognize numerous patterns characteristic of cell debris (damage-associated molecular patterns - DAMPs) or pathogens (pathogen-associated molecular patterns - PAMPs). Such receptors are toll-like receptors (TLRs) and NOD-like receptors (NLRs), which, upon recognition of their ligands, trigger intracellular cascades, affecting gene expression and production of cytokines. Pathogenic conditions, as in the case of diabetes and malignancy, alter these signaling pathways and shape macrophage inflammatory responses [4].

According to their inflammatory status, macrophages are classified as classically activated (pro-inflammatory, M1) and alternatively activated (anti-inflammatory, M2) macrophages, a classification also associated with distinct gene expression profiles [5]. Pro-inflammatory, M1 macrophages are known to initiate the acute phase of inflammation by producing pro-inflammatory cytokines, such as tumor necrosis factor alpha (TNFa), interleukin (IL)-6, IL-12, and IL- $1 \beta$, exhibiting increased oxidative burst, nitric oxide (NO) production, and antibacterial activity. On the contrary, M2 macrophages mediate antiparasitic immune responses, wound healing process, and resolution of inflammation by producing a range of anti-inflammatory molecules including TGF- $\beta$ and IL-10 and express arginase-1, Fizz1, and $\mathrm{Ym} 1$ [5]. Macrophage activation is a complex, tightly controlled process, which includes different intracellular signaling pathways $[1,3]$. Within each activation phenotype, macrophages may express different cell surface molecules and transcription factors or obtain epigenetic changes, depending on the activation stimulus [6]. These differences become more profound when translated in vivo, particularly in the context of multifactorial diseases, such as cancer and diabetes. Thus, the classical dichotomy of the macrophage phenotype is considered an oversimplified model with limited physiological relevance since studies reveal the existence of multiple macrophage populations expressing distinct profiles of pro- and anti-inflammatory genes [1].

Over the years, scientific interest has focused on studying macrophage responses at the level of signal transduc- tion and transcriptional regulation in response to TLR signals and how these contribute to different pathologies $[7,8]$. However, recent studies have highlighted that the macrophage inflammatory state is at a large extent regulated at the level of cell metabolism. Obtaining and maintaining an activation phenotype involves an array of energy-demanding biochemical processes. Macrophage ability to mount certain responses is defined by cell intrinsic factors, such as enzymatic activity, and extrinsic factors such as nutrient availability [9]. For that reason, cells reprogram their metabolism in order to fulfill energy requirements, and these bioenergetic rearrangements, in turn, dictate the macrophage phenotype and responses. Understanding macrophage metabolism can support identification of molecules that control macrophage responses and facilitate development of novel macrophagebased therapeutic strategies.

In this review, we present the current knowledge on metabolic regulation of macrophage activation. We first outline the metabolic routes that macrophages utilize in response to different TLR signals. We then describe how exacerbated glucose and insulin signaling in the context of diabetes alter macrophage metabolism and activation of phenotypes. Finally, we present the current knowledge on macrophage metabolism in tissue microenvironments. We focus on obesity and adipose tissue as well as tumors, being 2 conditions where macrophages play a critical role in disease pathogenesis.

\section{Metabolic Pathways Involved in Macrophage Activation by TLR Signals}

Macrophages have long been considered the sentinel cells of the innate immune system. As professional phagocytes, they sense, engulf, and successfully eliminate hazardous factors in order to maintain homeostasis [2]. Their proficient phagocytic capacity is attributed to various cell surface receptors, known as pattern recognition receptors (PRRs). The PRR superfamily includes both TLRs and NLRs, which allow cells to recognize numerous highly conserved PAMPs and DAMPs [3]. Upon ligand recognition, PRRs initiate signaling cascades that activate inflammatory responses. In both humans and mice, TLR subfamilies comprise several members that differ in terms of ligand recognition, localization, and signal transduction [10]. However, there is limited evidence on the metabolic rewiring that TLR signaling dictates and how these metabolic routes intervene with inflammatory gene expression and macrophage activation. 


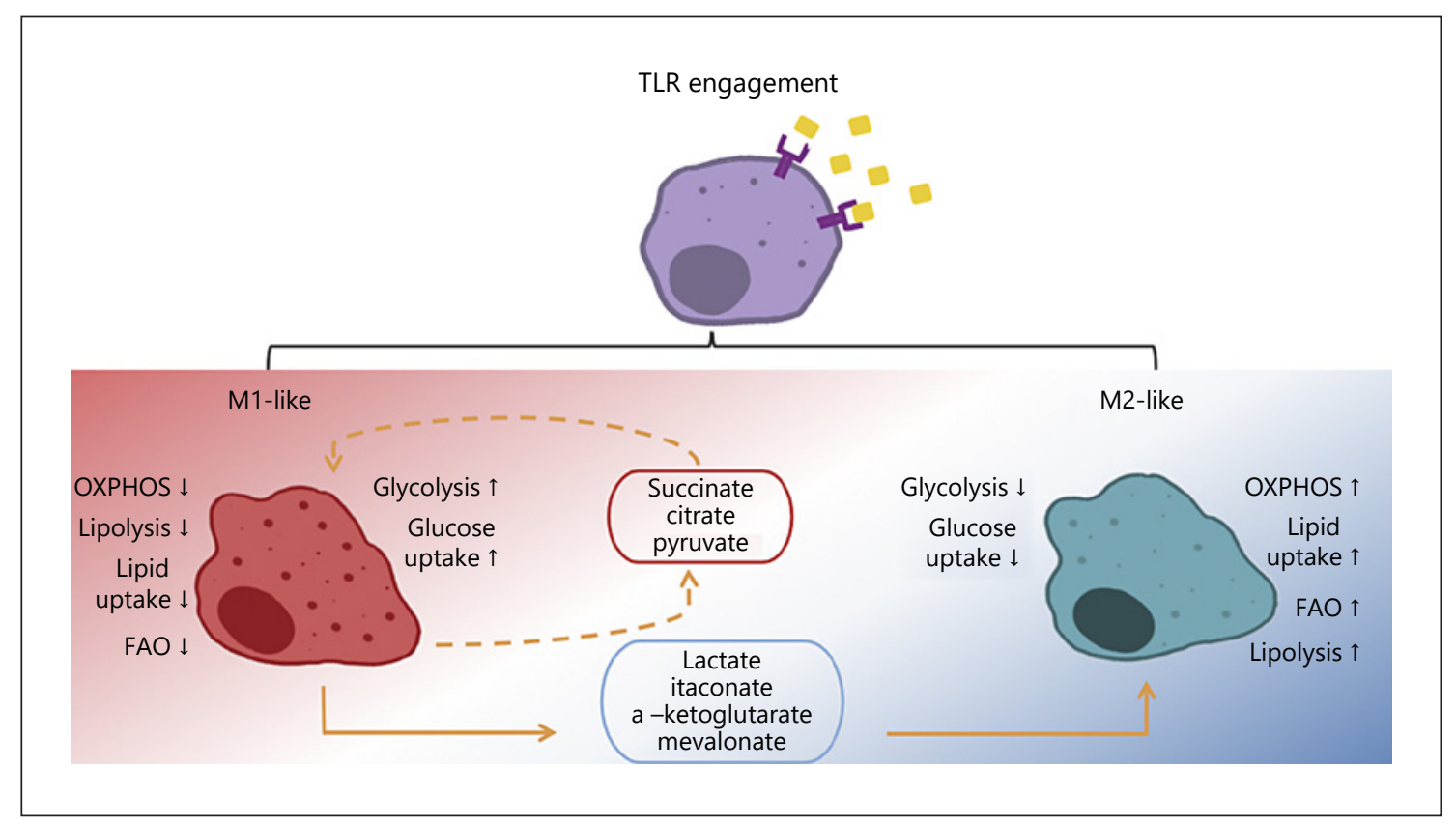

Fig. 1. Macrophage phenotype and metabolic rewiring upon TLR activation. TLR, toll-like receptors; OXPHOS, oxidative phosphorylation; FAO, fatty acid oxidation.

In macrophages, TLR stimulation initiates the expression of pro-inflammatory gene clusters, followed by the induction of anti-inflammatory markers at later time points. This transition from an M1-like to an M2-like state is supported by utilization of different energy sources and metabolic pathways. In vitro studies revealed that IFN $\gamma /$ LPS-activated macrophages (M1-like) display increased glycolytic burst and pentose phosphate pathway (PPP) flux that cause rapid energy production [11]. On the other hand, IL-4-induced macrophages (M2-like) rely their metabolism on more sustainable energy sources like mitochondrial respiration (oxidative phosphorylation [OXPHOS]) and fatty acid oxidation (FAO) [11]. Increased energy requirements at early time points following LPS stimulation are secured by maximal respiration, while at later time points, OXPHOS is reduced, and these changes are associated with time-dependent transcriptional signatures [12]. However, in different inflammatory conditions and TLR stimuli, macrophages obtain an array of activation phenotypes, each one of which is characterized by a distinct metabolic profile (Fig. 1).

\section{Glucose Metabolism in Response to TLR}

Glucose is the primary source of energy for macrophages. Upon TLR stimulation, macrophages shift towards increased glucose uptake and catabolism to meet their metabolic requirements, a shift highly conserved in metazoans $[13,14]$. Glycolysis starts with the uptake of glucose via specific glucose transporters. Glucose is then converted to glucose-6-phosphate (G6P), a reaction catalyzed by hexokinases (HKs). G6P can either be catabolized toward pyruvate, producing 2 ATP molecules, or enter PPP and fuel ribose and NADPH generation. Similarly, the pyruvate produced by anaerobic glycolysis can either be further metabolized to lactate or enter mitochondria [15]. There, it is converted into acetyl-CoA, the primary substrate of Krebs cycle (TCA cycle), used in OXPHOS for the production of 36 ATP molecules [16]. Although the glycolytic route is less effective than OXPHOS in terms of ATP production, it is an accelerated catabolic pathway that takes place in the cytosol, immediately fueling PPP and creating intermediate molecules useful for other pathways. Macrophages require large amounts of energy in a short time to respond to a noxious stimulus, and thus, glycolysis is a preferred source. As mentioned previously, classical macrophage activation by LPS/IFN $\gamma$ (M1-like) boosts glycolysis at the cost of OXPHOS, whereas IL-4/IL-13-stimulated cells (M2-like) use primarily OXPHOS for energy production $[14,16$, 17].

\section{Glycolysis}

TLR signaling elicits an M1-like macrophage response, characterized by a switch from OXPHOS to glucose up- 
take and anaerobic glycolysis [13, 18-23]. Glycolytic burst is tightly controlled by several enzymes that support the pro-inflammatory phenotype of macrophages. The first enzyme controlling the initial rate-limiting step in glycolysis is HK1. In addition to its role in converting glucose to G6P, HK1 is indispensable for pro-inflammatory responses like inflammasome activation. $\mathrm{HK} 1$ activity is regulated by the mechanistic target of rapamycin complex 1 (mTORC1), pharmacological inhibition of which suppresses HK1 expression, and pro-inflammatory cytokine production in TLR-stimulated macrophages [24, 25]. Upon sensing of TLR ligands, expression of PFKFB3 and $\alpha$-enolase is also induced [26-28]. Both enzymes skew macrophage polarization toward production of proinflammatory mediators, and their expression has been linked to autoimmune pathologies [26]. Pyruvate is the final product of glycolysis produced by pyruvate kinase M2 (PKM2), an enzyme that exerts distinct activities when forming dimers or tetramers, associated with glycolysis and gene transcription, respectively. In LPS-activated murine bone marrow-derived macrophages (BMDMs) dimerized PKM2 together with hypoxia-inducible factor 1 (Hifl $\alpha)$ and signal transducer and activator of transcription 3 (STAT3) license transcription of IL-1b, whereas tetrameric PKM2 upregulates anti-inflammatory IL-10 transcription $[29,30]$. Although most glycolytic enzymes support M1-like characteristics, glyceraldehyde-3-phosphate dehydrogenase (GAPDH) is known to possess both pro- and anti-inflammatory properties. At a resting state or at the state of endotoxin tolerance, GAP$\mathrm{DH}$ binds and downregulates TNFa expression at the posttranslational level [31]. Upon TLR stimulation and activation of glycolysis, the citrate-derived metabolite malonyl-CoA leads to malonylation of GAPDH, resulting in dissociation from TNFa mRNA, thus promoting its translation [32].

Studies using macrophages of human or murine origin have shown that macrophages challenged with TLR ligands and bacterial components exhibit increased an extracellular acidification rate (ECAR), indicative of lactate release, and a decreased oxygen consumption rate (OCR), indicative of mitochondrial activity [33]. This rerouting is accompanied by increased expression of glycolytic genes including Glut1, Hk1, and Pfkfb3, enhanced bactericidal activity and reactive oxygen species (ROS) production, and pro-inflammatory cytokine release [33]. Loss of glycolytic commitment by 2-deoxyglucose treatment abrogates the aforementioned phenotype, indicating that glycolysis is indispensable for M1-like macrophage activity $[34,35]$. However, under chronic and ex- acerbated TLR signaling, monocytes suffer dysfunctional glycolysis, resulting in reduced pro-inflammatory cytokine release [36].

TLR and cytokine signals also activate the PI3K/Akt/ mTOR pathway, a central regulator of cell metabolism. PI3K activates Akt, which in turn phosphorylates and inactivates the tumor suppressor complex 2 (TSC2). TSC2, along with TSC1, inactivates mTORC1. Thus, PI3K/AKT positively regulates mTORC1. Upon TLR stimulation, mTOR acts as a glucose sensor and reinforces the expression of Hif1a, a central regulator of glycolysis [35-37]. TLR signaling also induces nuclear factor kappa-B (NFkB)-mediated Hif1a expression. Hif1 $\alpha$ is a key regulator of glycolytic genes [38]. Pharmacological inhibition or depletion of Hifla in murine peritoneal macrophages blocks glycolysis at the transcriptional level and M1-like functions such as antibacterial/antifungal response, IL-6, $\mathrm{TNF} \alpha, \mathrm{IL}-1 \mathrm{~b}$, and ROS production $[35,38]$. Likewise, BMDMs treated with rapamycin, an inhibitor of mTORC1, display reduced lactate production, oxidative burst, and activity against pathogens [23]. Moreover, TLR4 stimulation in BMDMs prompts phosphorylation, activation, and mitochondrial translocation of the transcription factor STAT3, via TANK-binding kinase 1 (TBK-1). In mitochondria, STAT3 promotes both glycolysis and OXPHOS since inhibiting its phosphorylation leads to an impaired ECAR (succinate production) and OCR [39].

Macrophage metabolic rerouting toward glycolysis is orchestrated by an extensive network of epigenetic modifications. Class I and II histone deacetylases (HDACs) and specifically HDAC7 and HDAC3 control the expression and interact closely with glycolytic enzymes, thus licensing the glycolytic switch and pro-inflammatory burst $[12,40,41]$. However, other members of these families have opposing functions since global inhibition of HDACs promotes glycolysis and the M1-like phenotype in treated cells $[42,43]$. TLR-induced changes in macrophage histone acetylation are further supported by the activation of ATP citrate lyase that enriches such modifications, enhancing the production of pro-inflammatory mediators such as IL-6, IL-12b, and CCL1 [44]. Finally, lactate, the main by-product of exacerbated glycolysis in M1-like cells can act as an epigenetic regulator by promoting modification of histone lysine residues. Increased lactate concentrations are correlated with elevated lysine lactylation on M2 gene promoters, enhancing M2 gene expression $[45,46]$. 


\section{TCA Cycle}

Following TLR stimulation, increased glucose and lipid metabolism results in accumulation of intermediate metabolites that further alter macrophage metabolic routes $[39,44,47]$. Among those is pyruvate, the main product of exacerbated glycolysis in M1-like activated macrophages. In normoxia, pyruvate enters mitochondria, where pyruvate dehydrogenase $(\mathrm{PDH})$ oxidizes it to acetyl-CoA. However, under oxygen deprivation, as is the case at the inflammatory site, the stabilized Hifl $\alpha$ activates PDH kinase that inhibits PDH activity, boosting the inflammatory burst of macrophages [48]. Others report that TLR4 stimulation in RAW macrophages sustained $\mathrm{PDH}$ activity, supporting type I interferon and pro-inflammatory cytokine production [47]. Likewise, citrate and its conversion to acetyl-CoA by ATP-citrate lyase reinforces the M1-like phenotype by facilitating histone acetylation on pro-inflammatory gene promoters and subsequent upregulation of M1-like genes [44]. On the contrary, accumulation of a-ketoglutarate $(\mathrm{aKG})$ shifts macrophage metabolism toward utilization of FAO and interacts with JMJD3 histone demethylase to foster activation of M2-like transcription program [49]. Another important TCA cycle metabolite with signaling functions is succinate. LPS stimulation results in increased production of succinate which, among other functions, allows repurposing mitochondria to ROS production [50]. In LPS-activated macrophages, succinate conversion to fumarate by succinate dehydrogenase has been known to allow pro-inflammatory gene expression, inflammasome activation, and ROS production [50,51]. Succinate dehydrogenase activity and inflammatory burst are inhibited by itaconate. Itaconate is abundantly produced in TLRactivated macrophages and possesses anti-inflammatory activity since it blocks inflammasome activation and subsequent IL-1b and IL-18 release [52, 53]. Itaconate alkylates and inactivates KEAP1, an inhibitor of the transcription factor Nrf2, known to suppress inflammatory gene expression [54]. Another mechanism of anti-inflammatory action of the itaconate derivative 4-octyl itaconate is via alkylation of GAPDH and subsequent inhibition of glycolysis [55]. There is also evidence that NO regulates itaconate and directly inhibits glycolysis, skewing macrophages toward the acquisition of an alternative M2-like phenotype [56-58]. In addition to the TCA cycle, a parallel pathway equally important for the regulation of the inflammatory response of macrophages in response to TLR is that of mevalonate [59]. A recent study showed that upon TLR triggering, mevalonate promotes protein geranylgeranylation. Deficits in this pathway lead to up-

Metabolic Regulation in Macrophages regulation of pro-inflammatory cytokines and impairment of type I interferon and anti-inflammatory cytokine production [60].

\section{Oxidative Phosphorylation}

OXPHOS is also important for antibacterial responses and mainly supports resolution of inflammation; thus, stimulated macrophages utilize it for energy production at later stages of the inflammatory response [61]. The $\mathrm{PI} 3 \mathrm{~K} / \mathrm{Akt}$ axis has a pivotal role in OXPHOS regulation upon TLR stimulation. Specifically, LPS stimulation of BMDMs positively regulates the expression of the mitochondrial related genes Cox $I / I V$ and $T$-fam, as well as mitochondrial membrane potential. This phenotype depends on the PI3K/AKT pathway since $A k t 1^{-/-}$macrophages or BMDMs treated with chemical inhibitors of Akt displayed a robust pro-inflammatory phenotype, less mitochondrial activity, and impaired viability upon LPS stimulation [62]. TLR4-mediated regulation of OXPHOS is time-dependent; at early time points, it increases to maximal respiration, while at later time points, TLR4 signals suppress OXPHOS [38].

Although TLR4 signaling reroutes macrophages toward glycolysis while decreasing OXPHOS, this is not the case for all TLR ligands and complex pathogenic stimuli. When human monocytes are activated with TLR2 ligands or whole pathogen lysates, both glycolysis and OXPHOS are increased. Such upregulation of mitochondrial metabolism facilitates response to pathogens, while its inhibition blocks both phagocytosis and pro-inflammatory cytokine production [63].

\section{Lipid Metabolism in Response to TLR}

While TLR activation in macrophages shifts glucose metabolism toward glycolytic burst, changes in lipid metabolism are more complex [64]. Lipid metabolism is a system of finely tuned processes including lipid uptake, lipolysis, FAO, lipogenesis, and fatty acid (FA) synthesis, supporting the energy requirements of macrophage activation. Upon engulfment, circulating lipids are esterified and stored in characteristic lipid droplets (LDs) inside macrophages [65]. When cellular energetic demands dictate their breakdown, lipids are catabolized either enzymatically or via lysosomes, generating FAs and cholesterol [66]. Carnitine palmitoyltransferase then transports FAs to mitochondria, where they are metabolized to acetyl-CoA through a process known as FAO or $\beta$-oxidation [67].

In most cases, lipid metabolism in M1-like activated macrophages is skewed toward FA synthesis, storage, and 
production of intermediate derivatives that support expression of pro-inflammatory markers. Ablation of FA synthase attenuates macrophage responses involved in pro-inflammatory cytokine production in the context of diabetes, suggesting that endogenous FA synthesis regulates the macrophage phenotype, an effect mediated by changes in cell membrane composition [68]. On the contrary, M2-like macrophages utilize FAO to break down FAs and produce energy for anti-inflammatory purposes [64]. In fact, both murine and human IL-4-stimulated M2 macrophages display elevated lipolysis, FAO, and OXPHOS, compared with M0 and M1 cells. This metabolic route is vital for M2 cells since its genetic or pharmacological inhibition results in loss of M2-like characteristics such as CD206, CD301, PD-L2, and RELMa surface markers, as well as effective parasite elimination [66]. Accordingly, RAW macrophages express a constantly active form of CTP1A and thus have enhanced FAO, downregulated stress, and inflammatory responses [69]. Nevertheless, other studies support that the FAO pathway orchestrates the macrophage M1-like polarization status [70-73]. In LPS-stimulated BMDMs, NADPH oxidase 4-derived ROS regulates CPT1A and licenses FAO and NLRP3 inflammasome activation [63]. At the same time, FA crystals induce IL-1 a production by uncoupling mitochondrial respiration independent of inflammasome, contributing to atherosclerosis in mice $[72,73]$. Notably, IL-4-stimulated Cpt2 $2^{-/-}$macrophages, though incapable of metabolizing lipids via FAO, display characteristics of M2-like activation, indicating that in contrary to what it is believed, the M2-like phenotype may be independent of FAO commitment [71]. Thus, TLR signals can utilize multiple metabolic pathways to facilitate M2-like polarization.

Following TLR engagement, lipid biosynthesis and FA production are reduced, but they increase at later time points, supporting resolution of inflammation [74]. These changes are partly mediated by the histone deacetylases Sirt1 and Sirt6. In THP1 monocytes, LPS stimulation increasesSirt6andSirt1 that downregulate Hif1 $\alpha$-dependent glycolysis and boost lipid uptake and FAO, respectively [20]. Other central regulators of lipid metabolism in macrophages are the sterol regulatory element-binding proteins (SREBPs). In LPS-activated murine BMDMs, SREB$\mathrm{P} 1 \mathrm{a}$ acts downstream of mTORC1 and controls phagocytosis by modulating the lipid content of the cell membrane and the interactions with cytoskeletal components [75]. In TLR4-stimulated BMDMs and human MDMs, SREBP1 enhances lipid synthesis necessary for the anti-inflammatory shift of macrophages, by inducing epigenetic modi- fication and RNA polymerase recruitment on genes encoding enzymes critical for this biosynthetic pathway [74]. Independent studies indicate that SREBP1a also possesses pro-inflammatory activity since its depletion in macrophages distorts inflammasome activation and proinflammatory cytokine burst, upon challenge with bacteria [70]. SREBP2 controls cholesterol and lipid biosynthesis since Srebr $2^{-/}$BMDMs display reduced cholesterol synthesis, which correlates with increased cGAS-STINGTBK1-IRF3 signaling and type I IFN production [76]. Similarly, the nuclear factor liver X receptor (LXR) induces cholesterol efflux and suppresses pro-inflammatory activity in murine macrophages [77].

Although TLR stimulation induces universal changes in FA biosynthesis, as a shotgun lipidomic analysis revealed, different TLR ligands promote distinct patterns in macrophage lipid composition. These changes are dynamic through time and depend on the cellular lipid content and the activity of signal transduction molecules downstream of TLRs. Specifically, signal transduction via MyD88 upregulates long-chain FA synthesis, whereas TRIF signaling cascade activation suppresses long-chain FA synthesis and supports type I IFN production [10]. Accordingly, other studies demonstrate that during viral infection, TLR engagement with viral PAMPs diminishes sterol and FA synthesis to enhance the antiviral activity of macrophages [59, 76]. Specifically, TLR3 stimulation in macrophages augments lipid uptake while hindering cholesterol and FA synthesis, whereas induction of TLR9 increases intracellular accumulation of FAs and formation of LDs [40, 43]. In contrast to data obtained using a single TLR agonist, pathogen-sensing and concomitant infection alter macrophage metabolism toward increased lipid uptake, triglyceride storage, and lipolytic retardation [78-80].

Overall, TLR signaling orchestrates metabolic changes that are central for regulating inflammatory responses. The substrates that macrophages use as a source of energy and the metabolic pathways that they utilize determine their activation phenotype, type, and magnitude of the response.

\section{Insulin Signaling and Its Role in Macrophage Activation}

The effect of TLR signaling on macrophage metabolism and responses is affected by parallel signals that independently tune cell metabolism. Insulin signals regulate cell metabolism by controlling glucose uptake and 


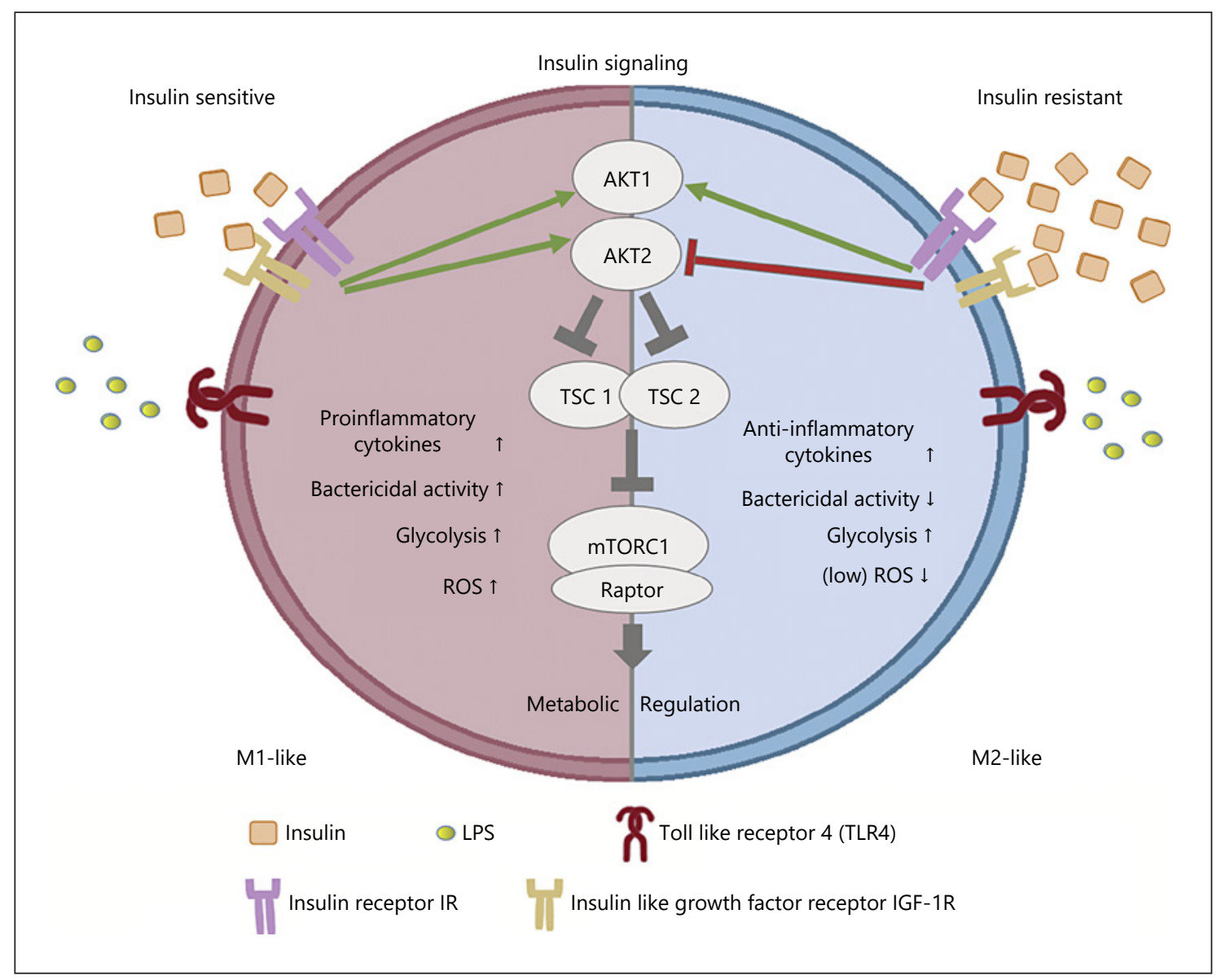

Fig. 2. Signaling and metabolic rewiring in insulin-sensitive and insulin-resistant macrophages. ROS, reactive oxygen species; mTORC1, mechanistic target of rapamycin complex 1.

metabolism (Fig. 2). Even though macrophages do not express the glucose transporter Glut4, the transporter that readily responds to insulin receptor (IR) signals to transfer glucose, and they express glucose transporters Glut1 and Glut3, which also provide glucose in response to IR and insulin-like growth factor 1 receptor (IGF1R) signals [81, 82]. Metabolic inflammation triggered by obesity is associated with a variety of factors that interfere with insulin signaling and may lead to insulin resistance. During the development of obesity, adipose tissue becomes inflamed and favors the shift of macrophage polarization from the predominant M2-like to M1-like phenotype. M1-like polarization is characterized by secretion of pro-inflammatory cytokines and tendency to form crown-like structures around the dead adipocytes for lipid scavenging [83, 84].

Early studies have shown that insulin modulates the antimicrobial capacity of monocytes/macrophages through enhancement of phagocytic ability and produc- tion of hydrogen peroxide but also alters their metabolism toward increased glucose and reduced glutamine metabolism [85]. In addition, insulin and IGF-1 induce TNFa synthesis in murine and human macrophages [86]. Moreover, glucose, insulin, and palmitate treatment of human monocyte-derived macrophages results in a metabolically activated phenotype, possessing both M1-like and M2-like characteristics [87]. Even though insulin stimulates glycolysis and facilitates activation and antimicrobial action of macrophages, continuous triggering from insulin allows development of resistance to its signals, resulting in insulin-resistant macrophages $[81,82]$. Insulin-resistant macrophages still utilize glucose but have reduced capacity to respond to inflammatory stimuli, possessing a distinct M2-like phenotype. This phenotype is associated with reduced antimicrobial capacity and may partly explain changes in innate immune responses observed in diabetic patients [81, 82]. 
Macrophages express all components of the insulin signaling cascade, indicating functional insulin signaling, but they can also develop insulin resistance in the context of systemic insulin resistance. Insulin mediates its signal through IR but also through its highly homologous IGF1R. Binding to its receptor results in subsequent activation of the PI3K/AKT pathway $[8,84]$. In animal models, myeloid-specific deletion of IR protects mice against HFD-induced obesity and promotes an anti-inflammatory signature of macrophages $[88,89]$. LysMCreIgf 1 r fl/fl mice, on the other hand, show increased adiposity and insulin resistance upon HFD feeding, suggesting that in the context of obesity, IGF1R is essential for suppression of inflammation [90]. Deletion of IGF1R on $A p o e^{-/-}$ background results in exacerbated pro-inflammatory responses, though functionally, macrophages display reduced phagocytic capacity [91]. Even though lack of IGF1R from macrophages results in exacerbation of inflammatory diseases such as obesity and atherosclerosis, in healthy mice, IGF1R-deficient macrophages exhibit defective insulin signaling and an M2-like phenotype accompanied by LPS hyporesponsiveness and defective pathogen clearance [82]. This discrepancy highlights that IGF1R in macrophages may differentially regulate responses to DAMP, TLR, or metabolite signaling in the context of different diseases. Myeloid-specific deletion of both IGF1R/IR receptors in mice protects against skin inflammation and causes attenuation of the pro-inflammatory and induction of the noninflammatory macrophage phenotype [92].

Insulin signaling through the Akt pathway has a crucial role in macrophage activation. The Akt2 isoform is the predominant isoform involved in insulin signaling. The absence of Akt2 from macrophages results in an M2-like polarization phenotype and insulin resistance. Macrophages that lack Akt2 express increased levels of the M2 polarization markers Arg1, Ym1, and Fizz1 and the transcription factor $C / E B P \beta$ [93]. In addition, genetic ablation of Akt2 results in upregulation of microRNA (miR)-146a that suppresses the TLR4 signaling pathway by targeting IRF5, TRAF6, and IRAK1 [94]. In human macrophages, LPS and other pro-inflammatory stimuli induce Akt2 activity and the production of cytokines that typify M1-like polarization [95]. Moreover, ablation of Akt2 from macrophages results in reduced atherosclerosis and foam cell formation in $\mathrm{Ldll}^{-1-}$ mice [96]. Akt promotes indirect activation of mTORC1 that is essential for insulin signaling propagation. In vivo and in vitro studies support the importance of $\mathrm{mTORC} 1$ in macrophage activation. Myeloidspecific deletion of the mTORC1-specific protein raptor elevates M2-like macrophage population in the adipose tissue of LysMCreRptorfl/fl mice, in the context of diet-induced obesity [97]. Accordingly, loss of TSC1 enhances LPS responses and favors M1-like polarization, while it suppresses the M2-like phenotype in response to IL-4 [98, 99]. However, there are also studies showing the lack of TSC1 results in both M1 and M2 polarization [100]. Furthermore, deletion of $T s c 1$ in myeloid cells protects mice from high fat diet-induced obesity, insulin resistance, and adipose tissue inflammation, via mTORC1-dependent M2-like macrophage polarization [101]. Sustained mTORC1 activation due to the lack of TSC2 in macrophages also enhances the expression of M2-like polarization markers [102]. Conversely, treatment with rapamycin, a suppressor of $\mathrm{mTORC1}$, promotes an M1-like phenotype, both in human and murine macrophages and blocks the anti-inflammatory potency of glucocorticoids in myeloid immune cells via increased JNK and NF- $\kappa B$ activation [103]. Overall, the AKT/TSC/mTORC1 signaling cascade dictates metabolic changes in macrophages in response to exogenous signals including those initiated by insulin, shaping the magnitude and type of responses.

\section{Macrophage Metabolism and Activation during Obesity}

Adipose tissue macrophages (ATMs) contribute to chronic low-grade inflammation, obesity-driven insulin resistance, and metabolic disease [104, 105]. During obesity, an increase in macrophage population in the adipose tissue is associated with a switch from an anti-inflammatory (M2-like) to an inflammatory (M1-like) phenotype, potentially as part of a mechanism to maintain metabolic homeostasis [106].

The increased number of ATMs is either due to the recruitment of circulating monocytes or due to local proliferation observed predominantly around dead adipocytes, at sites known as crown-like structures [107]. Scavenging of adipocyte debris from ATMs, in an attempt to process excessive amounts of lipids and avoid tissue hypoxia and lipotoxicity, is compromised in obesity. Additional protective functions of ATMs, including oxidative stress handling, are also insufficient, resulting in dysfunctional obese adipose tissue [108].

Metabolic reprogramming of macrophages is linked to their function [104]. Recently, the use of advanced technologies revealed a plethora of ATM subpopulations characterized by distinct metabolic and activation signatures depending on their location in the adipose tissue [109]. 
Lipid Metabolism in ATMs

ATMs from adipose tissue acquire a unique metabolically activated phenotype termed as "MMe" that exhibit mixed characteristics from M1-like type since they secrete pro-inflammatory mediators but also from M2-like cells [87]. The MMe metabolic phenotype is characterized by lipid catabolism, storage, efflux, and utilization. Exposure of ATMs in a lipid-rich environment drives the induction of the transcription factor peroxisome proliferator-activated receptor $\gamma$ (PPAR $\gamma)$ and the autophagyassociated protein $\mathrm{p} 62$, both associated with the M2-like phenotype [87]. These factors promote expression of the cholesterol efflux transporter ATP-binding cassette subfamily A member 1, the scavenger receptor CD36 that mediates uptake of lipids released from adipocytes undergoing lipolysis, and the LD-associated protein PLIN2 in MMe ATMs [87]. Induction of these genes is part of the attempt of the organism to buffer excessive lipid circulation and limit inflammation [110].

Lysosomal-dependent lipid metabolism possesses a crucial role in ATMs in obesity. Lysosomes participate in lipophagy, a process that regulates cholesterol efflux via LD degradation but also in the formation of an acidic compartment that allows the hydrolysis and internalization of adipocyte debris, a process called exophagy [111, 112]. Large amounts of lipids enter the cell and are delivered to lysosomes for degradation and catabolic processing. MMe ATMs have increased lysosomal biogenesis and lysosomaldependent lipid catabolism, characterized by enhanced expression of lysosomal proteins, including the structural lysosome protein LAMP2 and the acid lipase LIPA [113].

MMe ATMs display reduced LD formation, subsequently interfering with the final steps of autophagosome-lysosome fusion in lipophagy and/or lysosomal degradation cascade. Chemical or siRNA-mediated inhibition of autophagy through inhibition of $v A T P a s e$ or Lamp-1 expression leads to increased LD accumulation in macrophages [113-115].

Single-cell RNA sequencing analysis of ATMs revealed a $\mathrm{CD}^{+}$macrophage population that contains large amounts of intracellular lipids, is localized in crown-like structures, and secretes exosomes. $\mathrm{CD}^{+}$ATMs are characterized by upregulation of genes associated with lipid metabolism (Lpl and Plin2), lysosomal biogenesis, and activation (Acp5, Ctss, Lamp2, and Lipa) and expression of pro-inflammatory factors (Il1 $\alpha, I l 18$, Tnfo, and Ccl2), defining a signature of metabolic activation and lysosomal-dependent lipid metabolism [106].

A novel lipid-associated macrophage subset $\left(\mathrm{CD}^{+} \mathrm{CD}^{+} 3^{+}\right)$is expanded in obese adipose tissue, char- acterized by Trem2-driven gene expression profile involved in phagocytosis, lipid catabolism, and OXPHOS [116]. This macrophage subset expresses genes related to immune suppression (Lgals1 and Lgals3). Trem2-deficient macrophages display reduced expression of Lipa (lysosomal function), Cd36, Lpl, Fabp4, and Fabp5 (known to be involved in lipid metabolism) and reduced recruitment to crown-like structures [116]. Genetic ablation of Trem 2 in high fat diet-fed mice results in exacerbated weight gain, inflammation, and glucose intolerance [116].

\section{Glycolysis and OXPHOS in ATMs}

In the context of obesity, ATMs exhibit increased glycolysis and OXPHOS, as demonstrated by transcriptomics and extracellular flux analyses [117]. This increase depends on adipose tissue-derived factors [117]. Similar metabolic profile with increased glycolysis and OXPHOS is observed in human macrophages isolated from adipose tissue of obese individuals with T2D and is associated with a pro-inflammatory phenotype [117].

Oxidative stress is increased during obesity. Increased levels of ROS and nitrogen species result in the formation of oxidation-derived DAMPs (oxidized phospholipids) [118]. Oxidized phospholipids are found on oxidized LDL and on apoptotic cell membranes and are recognized by macrophages promoting either an antioxidant or proinflammatory phenotype. ATMs from obese adipose tissue acquire an M1/M2 hybrid phenotype CD $11 \mathrm{c}^{+} \mathrm{CD} 206^{+}$, exhibiting both pro- and anti-inflammatory characteristics and a highly activated metabolism, associated with increased respiratory capacity and glycolysis [119]. In contrast, ATMs from lean adipose tissue show a suppressed bioenergetic profile redirecting glucose metabolism and TCA metabolites to produce glutathione, essential for antioxidant function [118].

\section{Metabolic Changes in Peripheral Macrophages in the Context of Obesity}

In the periphery, macrophages from obese mice exhibit induction of glycolysis, as indicated by increased ECAR and expression of glycolytic genes (including Glut1, Glut3, Hk3, and Ldha). Inhibition of glycolysis, using 2-deoxyglucose abolished the M2-like phenotype of these cells [82]. Lipid-laden peritoneal macrophages isolated from $\mathrm{Ldlr}^{-/-} \mathrm{HFD}$-induced hypercholesterolemic mice show decreased mitochondrial mass and maximal respiration and disturbed abundance of several metabolites including itaconate [120]. In addition, reduced flux through the PPP contributes to impaired inflammatory 
responses and ROS production of foam cells during hypercholesterolemia [120].

Foam cells also display impaired cholesterol biosynthesis associated with suppression of Dhcr24 (24-dehydrocholesterol reductase), an enzyme that converts desmosterol into cholesterol [121]. Downregulation of Dhcr 24 results in accumulation of desmosterol that activates LXR and SREBP target genes, interferes with FA metabolism, and contributes to impaired inflammatory responses in macrophages from hypercholesterolemic mice [121]. Peritoneal macrophages from the diet-induced obesity model do not display foam cell characteristics, although they exhibit downregulation of several enzymes of the cholesterol biosynthesis pathway, including Dhcr24 [122]. Accumulation of desmosterol in macrophages from diet-induced obesity mice is also associated with upregulation of LXR and SREPB target genes and downregulation of inflammatory genes [122].

Overall, both glucose and lipid metabolism are altered in macrophages in the context of obesity, shaping the inflammatory phenotype. In the adipose tissue, ATM metabolism is crucial for the development of insulin resistance, and several signaling pathways are involved, highlighting the importance of the local microenvironment in modulating the macrophage phenotype and responses. Thus, in the context of obesity, the adipose tissue microenvironment differs from this in the aorta and in peripheral circulating macrophages and so does their phenotype and cell metabolism. For example, adipose tissue macrophages obtain a pro-inflammatory phenotype contributing to adipose tissue inflammation, while peripheral macrophages obtain an M2-like phenotype, contributing to foam cell formation and altered responses to pathogens $[81,82]$.

\section{Macrophage Metabolism in the Tumor Microenvironment}

Tumors are multicellular systems of high complexity and diversity, characterized by unique conditions. They embrace various types of cells, broadly classified into 3 categories: highly proliferative malignant cells, stromal, and immune cells. Inside the tumor microenvironment (TME), cells synergize and coordinate molecular processes that dictate tumor fate regarding growth, progression, immune evasion, and metastasis. The nature of TME affects the metabolism of participating cells but is also regulated by their metabolic by-products, and this dynamic communication is preserved throughout tumorigenesis.
Tumor progression is accompanied by exacerbated hypoxic conditions, nutrient scarcity, and accumulation of several cancer cell-derived metabolites and metabolic byproducts.

Inside the intricate TME, tumor-associated macrophages (TAMs) play an important role in shaping its landscape. According to their inflammatory signature, TAMs possess either M1-like or M2-like characteristics, and these contribute to the tumor phenotype and outcome [6]. In many types of cancers, M2-like TAMs have been associated with disease progression, whereas M1like TAMs mainly possess antitumoral activity, although some cases indicate that they favor cancer progression [123-126]. TAM polarization is controlled by metabolism that changes according to TME conditions.

\section{Lipid Metabolism in TAMs}

TAMs utilize FAO as a source of energy by expressing a repertoire of scavenger receptors that facilitate lipid uptake [65]. In several human cancers and in murine models of cancer, lipid accumulation in TAMs is mediated by CD36. According to single cell data analysis, CD36 is elevated in tumor myeloid cells, where it assists lipid uptake and catabolism via FAO, thus inducing TAM promalignant activity $[66,127]$. Following CD36 uptake, lysosomal lipolysis of FAs is critical for M2-like function of TAMs [66]. In addition to scavenger receptors, several other mechanisms facilitate lipid uptake in TAMs. TAMs from hepatocellular carcinoma express low levels of RIPK3 kinase, which supports PPAR $\gamma$ activation via caspase 1 , promoting FA metabolism and anti-inflammatory signals [128]. Another study supported that PPAR $\gamma$ cleavage by caspase 1 regulates lipid accumulation in TAMs. Truncated PPAR $\gamma$ deactivates MCAD enzyme, leading to FAO inhibition, TAM reprogramming, and cancer progression [129]. As observed in many cancers, increased lipid accumulation in TAMs is attributed to reduction of monoacylglycerol lipase activity. Downregulation of monoacylglycerol lipase suppresses M1-like characteristics and offers tumor progression and immunosurveillance [130]. Accordingly, unsaturated FA uptake in TAMs supports their alternative activation by reinforcing mitochondrial respiration and LD formation [65]. Increased LD content in TAMs correlates with tumor formation in colorectal cancer patients, whereas inhibition of $\mathrm{LD}$ formation and degradation restrains tumor growth [65]. Moreover, utilization of LDs as energy source activates the mTORC2-IRF4 axis involved in M2-like polarization of TAMs $[65,131]$.
60

J Innate Immun 2022;14:51-67

DOI: $10.1159 / 000516780$ 


\section{Glucose Metabolism in TAMs}

Due to increased cell proliferation, many nutrients, including glucose, are scarce in the TME, leading to competition for energy resources. In these glucose-deprived conditions, TAMs adjust their metabolism to match that of cancer cells by increasing glucose uptake and glycolytic activity. Although glycolysis is primarily associated with M1-like responses, at the site of tumor, M2-like TAMs also use glycolysis for fulfilling their energy demands [131]. A recent comprehensive proteomic analysis revealed that TAMs isolated from breast tumors have increased expression of HK2 and several other factors involved in glycolysis, explaining their mixed phenotype characteristics [132]. The Warburg effect in TAMs of pancreatic tumors contributes to metastasis through increased VEGF and TGF- $\beta$ production. Disruption of the glycolytic pathway results in reversal of the pro-tumoral phenotype of TAMs [133]. However, other studies support that in oxygen-deprived conditions, TAMs antagonize endothelial cells for the same energy source, glucose [7]. Increased glucose uptake and glycolysis in TAMs hinder EC hyperactive status, preventing vascularization, cancer cell leakage, and metastasis. In contrast regulated in development and in DNA damage response 1 (REDD1) inhibition of mTORC1-mediated glycolysis augments tumor-invasiveness [7, 134].

\section{Amino Acid Metabolism in TAMs}

In addition to glucose and lipid metabolism, amino acids (AAs) play an important role in survival and energy production in TAMs. AA availability enhances the M2like phenotype, whereas protein, and specifically methionine and cysteine, restriction rewires TAM responses toward the M1-like phenotype. In combination with immunotherapy, AA restriction significantly reduces tumor growth in mouse models of prostate and renal cancer [135]. Glutamine is another crucial AA for both cancer cells and immunosuppressive TAMs [136]. RNA profiling of TAMs isolated from a triple-negative breast cancer model treated with a glutamine antagonist revealed that blockage of glutamine metabolism promotes M1-like characteristics. Glutamine restricts myeloid-derived suppressor cell differentiation toward tumoricidal TAMs, whereas its inhibition enriches pro-inflammatory TAMs at the tumor site in a mouse model of breast cancer [137]. In addition to that, M2-like TAMs have increased expression of glutamine synthase (GS) and production of glutamine. Both GS and glutamine are crucial for the immunosuppressive and angiogenic functions of TAMs since their depletion shifts TAMs to a pro-inflammatory and immunostimulatory phenotype [138]. Arginine metabolism is also fundamental for TAM function and tumor progression being the substrate for several immunomodulatory molecules. Arginine is the substrate for NO synthase and arginase that skew macrophage metabolism toward M1-like and M2-like phenotypes, respectively. Many studies support that pro-tumoral TAM function associates with enhanced (ARG1) and diminished iNOS activity. The absence of Arg1 expression in vivo leads to limited tumor growth, by enhancing M1-like cells in mouse models of ovarian cancer or melanoma $[139,140]$. By-products of AA and protein metabolism can also impact TAM function. Retinoic acid derived from vitamin A catabolism in malignant cells is known to enhance monocyte differentiation toward pro-tumoral TAMs [141]. Branched-chain AAs (BCAAs) like leucine, isoleucine, and valine skew macrophages toward a pro-tumoral M2-like fate indirectly. In myeloid leukemia and glioblastoma, malignant cells consume BCAAs and catabolize them into branched-chain ketoacids [142, 143]. Branchedchain ketoacids are then uptaken by TAMs, where they inhibit phagocytosis, suppressing antitumor activity [142].

\section{TCA Cycle in TAMs}

Glycolysis, FAO, and AA metabolism fuel TAM Krebs cycle with substrates for ATP production. Much like LPSstimulated macrophages, TAMs display dysregulation of the TCA cycle and accumulation of intermediate metabolites, responsible for TAM localization and function and overall TME remodeling [144]. Lactate is a major byproduct of cancer cell glycolytic activity. When uptaken by TAMs, lactate acts via stabilizing Hifl $\alpha$ and promotes the expression of M2 related genes, suppressing immunosurveillance and promoting tumor growth [45, 145]. However, under intense hypoxic conditions, high lactate concentrations promote TAM apoptosis [144]. Succinate is another metabolic intermediate that accumulates in TAMs. A recent study demonstrated that lung cancer patients have high levels of succinate in the serum, primarily produced by cancer cells [67]. In LPS-induced macrophages, succinate production confers a danger signal causing HIF1 $\alpha$ and IL- $1 \beta$ upregulation [51]. In TAMs however, succinate uptake activates the PI3K-HIF1 $\alpha$ axis, resulting in an M2-like status [67]. aKG, a metabolite profoundly produced via glutaminolysis, has a similar effect in macrophage activation. Even though a high aKG/succinate ratio in pancreatic ductal adenocarcinoma cells associates with premalignant gene expression and prevention of tumor aggressiveness, in TAMs, the same ratio 
induces anti-inflammatory activity and tumor growthpromoting activity [146, 147]. Finally, itaconic acid emerges as a critical orchestrator of macrophage responses. Macrophage treatment with itaconate leads to the arrest of inflammasome activation, and ROS and pro-inflammatory cytokine production, while enhancing lactate production and consequently intracellular succinate accumulation [53]. In the context of tumor-TAM interaction, itaconate drives macrophage metabolic rewiring toward increased OXPHOS and aerobic glycolysis, further promoting tumor progression [148].

\section{The Effect of TME in TAM Metabolism}

The metabolic route and responsiveness of TAMs are modulated by several factors prevailing in TME. Hypoxia is a central force for cancer progression and immune surveillance $[149,150]$. Hypoxic conditions boost the expression and stabilization of both HIF1 $\alpha$ and HIF $2 \alpha$, critical regulators of glycolysis, skewing the metabolic fate of TAMs toward the anaerobic glucose catabolism (Warburg effect) $[47,51,138,150]$. In gastric cancer, hypoxia induces TAM M2-like function by rerouting metabolism toward glycolysis via the HIF1a-REDD1 axis [134]. Hypoxic conditions have also been linked to diminished antigen presentation and increased mitochondrial stress that educate TAMs towards cancer surveillance [151, $152]$.

Cancer-derived vesicles and their cargo are also fundamental in shaping TAM metabolism and function. Such vesicles carry proteins, transcription factors, and miRs that further promote the M2-like status of TAMs $[139,153,154]$. Colon cancer cells produce exosomes that carry miR-1246 and lncRNA RPPH1, supporting TAM differentiation $[125,155]$. However, cancer-derived vehicles can also skew macrophage polarization toward M1-like state by suppressing FAO and activating the Akt/ mTOR pathway $[156,157]$.

Another critical component of TME affecting TAM function is the concentration of ROS. Although ROS are designated markers of the M1-like phenotype, they are crucial for M2-like polarization of TAMs. When sensed by TAMs, ROS reprogram the macrophage transcriptional profile toward immune suppression and angiogenesis [158]. They also mediate the TAM M2-like phenotype by inducing phosphorylation and activation of STAT3. Treatment of macrophages with ROS or STAT3 inhibitors suppresses their M2-like activation status [159]. Mitochondria-derived ROS promote stabilization of Hif $1 \alpha$, suppressing mitochondrial respiration of TAMs and enhancing their glycolytic commitment [160].
Overall, a variety of factors present in the TME dictate the phenotype of macrophages in a way that enhances their tumor-promoting activity. Metabolic changes arising from secondary metabolite signaling are hallmarks of this phenotype. Hence, modulating TAM metabolism, in conjunction with modulating tumor cell metabolism, can be a promising anticancer therapeutic approach.

\section{Conclusions}

Macrophages require energy to mount an effective innate immune response. As highly plastic cells, they utilize variable energy sources and activate downstream metabolic pathways like glycolysis, FAO, and AA metabolism for energy production. Increased glycolytic burst and PPP flux support acute energy production and pro-inflammatory responses, whereas OXPHOS and FAO provide sustained energy for macrophage anti-inflammatory functions. Macrophage metabolism and its modulation is determined by environmental signals like nutrient and oxygen abundance, pathogenic signals, and nonpathogenic factors, such as insulin and secondary metabolites. The net result is that responses to pathogenic or nonpathogenic TLR signals vary and described by the different activation phenotypes, being key determinants of associated pathologies. It is, therefore, clear that targeting macrophage metabolism can modulate their function and provide therapeutic solutions for multiple diseases.

\section{Conflict of Interest Statement}

The authors have no conflicts of interest to declare.

\section{Funding Sources}

The work was supported by the Hellenic Foundation for Research and Innovation grant (HFRI, General Secretariat for Research and Technology, GSRT Grant No. 1010) by the EU Commission and BBI-JU Horizon H2020; AQUABIOPRO-FIT project (grant number 790956) and cofinanced by the European Union and Greek national funds through the Operational Program Competitiveness, Entrepreneurship and Innovation under the call RESEARCH - CREATE INNOVATE, project code: T1E $\Delta \mathrm{K}-03846$, Acronym: iFUNF; and the research project CMBR (MIS 5002670) in the framework of the National Roadmap for Research Infrastructures.

\section{Author Contributions}

O.K., E.I., E.V., and C.T. drafted and reviewed the manuscript.
62

J Innate Immun 2022;14:51-67 DOI: $10.1159 / 000516780$ 


\section{References}

1 Murray PJ, Allen JE, Biswas SK, Fisher EA, Gilroy DW, Goerdt S, et al. Macrophage activation and polarization: nomenclature and experimental guidelines. Immunity. 2014 Jul; 41(1):14-20.

2 Gordon S, Taylor PR. Monocyte and macrophage heterogeneity. Nat Rev Immunol. 2005 Dec;5(12):953-64.

3 Glass CK, Natoli G. Molecular control of activation and priming in macrophages. Nat Immunol. 2016 Jan;17(1):26-33.

4 Russell DG, Huang L, VanderVen BC. Immunometabolism at the interface between macrophages and pathogens. Nat Rev Immunol. 2019 May;19(5):291-304.

5 Orecchioni M, Ghosheh Y, Pramod AB, Ley K. Macrophage polarization: different gene signatures in M1(LPS+) vs. classically and M2(LPS-) vs. alternatively activated macrophages. Front Immunol. 2019 May; 10:1084.

6 Vergadi E, Ieronymaki E, Lyroni K, Vaporidi $\mathrm{K}$, Tsatsanis C. Akt signaling pathway in macrophage activation and $\mathrm{M} 1 / \mathrm{M} 2$ polarization. J Immunol. 2017 Feb;198(3):1006-14.

7 Wenes M, Shang M, Di Matteo M, Goveia J, Martín-Pérez R, Serneels J, et al. Macrophage metabolism controls tumor blood vessel morphogenesis and metastasis. Cell Metab. 2016 Nov;24(5):701-15.

8 Guo S. Insulin signaling, resistance, and metabolic syndrome: insights from mouse models into disease mechanisms. J Endocrinol. 2014 Feb;220(2):T1-23.

9 Jha AK, Huang SC-C, Sergushichev A, Lampropoulou V, Ivanova Y, Loginicheva E, et al. Network integration of parallel metabolic and transcriptional data reveals metabolic modules that regulate macrophage polarization. Immunity. 2015 Mar;42(3):419-30.

10 Hsieh W-Y, Zhou QD, York AG, Williams KJ, Scumpia PO, Kronenberger EB, et al. Toll-like receptors induce signal-specific reprogramming of the macrophage lipidome. Cell Metab. 2020 Jul;32(1):128-43.e5.

11 Van den Bossche J, O'Neill LA, Menon D. Macrophage immunometabolism: where are we (going)? Trends Immunol. 2017 Jun;38(6): 395-406.

12 Mullican SE, Gaddis CA, Alenghat T, Nair MG, Giacomin PR, Everett LJ, et al. Histone deacetylase 3 is an epigenomic brake in macrophage alternative activation. Genes Dev. 2011 Dec;25(23):2480-8.

13 Krejčová G, Danielová A, Nedbalová $P$, Kazek M, Strych L, Chawla G, et al. Drosophila macrophages switch to aerobic glycolysis to mount effective antibacterial defense. Elife. 2019 Oct; 8:e50414.

14 Rodríguez-Prados J-C, Través PG, Cuenca J, Rico D, Aragonés J, Martín-Sanz P, et al. Substrate fate in activated macrophages: a comparison between innate, classic, and alternative activation. J Immunol. 2010;185(1):60514.
15 Soto-Heredero G, Gómez de las Heras MM, Gabandé-Rodríguez E, Oller J, Mittelbrunn M. Glycolysis: a key player in the inflammatory response. FEBS J. 2020 Aug;287(16): 3350-69.

16 Wang F, Zhang S, Vuckovic I, Jeon R, Lerman A, Folmes CD, et al. Glycolytic stimulation is not a requirement for M2 macrophage differentiation. Cell Metab. 2018 Sep;28(3):463-75. e4.

17 Wang F, Zhang S, Jeon R, Vuckovic I, Jiang X, Lerman A, et al. Interferon gamma induces reversible metabolic reprogramming of M1 macrophages to sustain cell viability and proinflammatory activity. EBioMedicine. 2018 Apr;30:303-16.

18 Yamada KJ, Heim CE, Xi X, Attri KS, Wang $\mathrm{D}$, Zhang W, et al. Monocyte metabolic reprogramming promotes pro-inflammatory activity and Staphylococcus aureus biofilm clearance. PLoS Pathog. 2020 Mar;16(3):e1008354.

19 Quero L, Hanser E, Manigold T, Tiaden AN, Kyburz D. TLR2 stimulation impairs anti-inflammatory activity of M2-like macrophages, generating a chimeric M1/M2 phenotype. Arthritis Res Ther. 2017;19(1):245-13.

20 Liu TF, Vachharajani VT, Yoza BK, McCall CE. NAD+-dependent sirtuin 1 and 6 proteins coordinate a switch from glucose to fatty acid oxidation during the acute inflammatory response. J Biol Chem. 2012 Jul;287(31): 25758-69.

21 Tan Z, Xie N, Banerjee S, Cui H, Fu M, Thannickal VJ, et al. The monocarboxylate transporter 4 is required for glycolytic reprogramming and inflammatory response in macrophages. J Biol Chem. 2015 Jan;290(1):46-55.

22 Van den Bossche J, Baardman J, Otto NA, van der Velden S, Neele AE, van den Berg SM, et al. Mitochondrial dysfunction prevents repolarization of inflammatory macrophages. Cell Rep. 2016 Oct;17(3):684-96.

23 Fensterheim BA, Young JD, Luan L, Kleinbard RR, Stothers CL, Patil NK, et al. The TLR4 agonist monophosphoryl lipid A drives broad resistance to infection via dynamic reprogramming of macrophage metabolism. J Immunol. 2018 Jun;200(11):3777-89.

24 Moon J-S, Hisata S, Park M-A, DeNicola GM, Ryter SW, Nakahira K, et al. mTORC1-induced HK1-dependent glycolysis regulates NLRP3 inflammasome activation. Cell Rep. 2015 Jul;12(1):102-15.

25 Eddie Ip WK, Hoshi N, Shouval DS, Snapper S, Medzhitov R. Anti-inflammatory effect of IL-10 mediated by metabolic reprogramming of macrophages. Science. 2017 May; 356(6337):513-9.

26 Bae S, Kim H, Lee N, Won C, Kim H-R, Hwang $Y$, et al. a-Enolase expressed on the surfaces of monocytes and macrophages induces robust synovial inflammation in rheumatoid arthritis. J Immunol. 2012 Jul;189(1): 365-72.
27 Tawakol A, Singh P, Mojena M, PimentelSantillana M, Emami H, MacNabb M, et al. HIF- $1 \alpha$ and PFKFB3 mediate a tight relationship between proinflammatory activation and anerobic metabolism in atherosclerotic macrophages. Arterioscler Thromb Vasc Biol. 2015 Jun;35(6):1463-71.

28 Wang Z, Kong L, Tan S, Zhang Y, Song X, Wang $\mathrm{T}$, et al. Zhx2 accelerates sepsis by promoting macrophage glycolysis via Pfkfb3. J Immunol. 2020 Apr;204(8):2232-41.

29 Palsson-McDermott EM, Curtis AM, Goel G, Lauterbach MAR, Sheedy FJ, Gleeson LE, et al. Pyruvate kinase M2 regulates Hif- $1 \alpha$ activity and IL- $1 \beta$ induction and is a critical determinant of the warburg effect in LPS-activated macrophages. Cell Metab. 2015 Jan;21(1):6580.

30 Xie M, Yu Y, Kang R, Zhu S, Yang L, Zeng L, et al. PKM2-dependent glycolysis promotes NLRP3 and AIM2 inflammasome activation. Nat Commun. 2016 Dec;7(1):13280.

31 Millet P, Vachharajani V, McPhail L, Yoza B, McCall CE. GAPDH binding to TNF- $\alpha$ mRNA contributes to posttranscriptional repression in monocytes: a novel mechanism of communication between inflammation and metabolism. J Immunol. 2016 Mar;196(6): 2541-51.

32 Galván-Peña $S$, Carroll RG, Newman C, Hinchy EC, Palsson-McDermott E, Robinson EK, et al. Malonylation of GAPDH is an inflammatory signal in macrophages. Nat Commun. 2019 Dec;10(1):338.

33 Haschemi A, Kosma P, Gille L, Evans CR, Burant CF, Starkl P, et al. The sedoheptulose kinase CARKL directs macrophage polarization through control of glucose metabolism. Cell Metab. 2012 Jun;15(6):813-26.

34 Fleetwood AJ, Lee MKS, Singleton W, Achuthan A, Lee M-C, O'Brien-Simpson NM, et al. Metabolic remodeling, inflammasome activation, and pyroptosis in macrophages stimulated by Porphyromonas gingivalis and its outer membrane vesicles. Front Cell Infect Microbiol. 2017 Aug;7:351.

35 Li C, Wang Y, Li Y, Yu Q, Jin X, Wang X, et al. HIF1a-dependent glycolysis promotes macrophage functional activities in protecting against bacterial and fungal infection. Sci Rep. 2018 Dec;8(1):3603.

36 Cheng S-C, Scicluna BP, Arts RJW, Gresnigt MS, Lachmandas E, Giamarellos-Bourboulis EJ, et al. Broad defects in the energy metabolism of leukocytes underlie immunoparalysis in sepsis. Nat Immunol. 2016 Apr;17(4):40613.

37 He L, Gomes AP, Wang X, Yoon SO, Lee G, Nagiec MJ, et al. mTORC1 promotes metabolic reprogramming by the suppression of GSK3-dependent Foxk1 phosphorylation. Mol Cell. 2018 Jun;70(5):949-60.e4. 
38 Cheng S-C, Quintin J, Cramer RA, Shepardson KM, Saeed S, Kumar V, et al. mTOR- and HIF-1 $\alpha$-mediated aerobic glycolysis as metabolic basis for trained immunity. Science. 2014 Sep;345(6204):1250684.

39 Balic JJ, Albargy H, Luu K, Kirby FJ, Jayasekara WSN, Mansell F, et al. STAT3 serine phosphorylation is required for TLR4 metabolic reprogramming and IL-1 $\beta$ expression. Nat Commun. 2020 Dec;11(1):3816.

40 Das Gupta K, Shakespear MR, Curson JEB, Murthy AMV, Iyer A, Hodson MP, et al. Class IIa histone deacetylases drive toll-like receptor-inducible glycolysis and macrophage inflammatory responses via pyruvate kinase M2. Cell Rep. 2020 Feb;30(8):2712-28.e8.

41 Ghiboub M, Zhao J, Li Yim AYF, Schilderink R, Verseijden C, van Hamersveld PHP, et al. HDAC3 mediates the inflammatory response and LPS tolerance in human monocytes and macrophages. Front Immunol. 2020 Oct;11: 550769.

42 Cox DJ, Coleman AM, Gogan KM, Phelan JJ, Ó Maoldomhnaigh C, Dunne PJ, et al. Inhibiting histone deacetylases in human macrophages promotes glycolysis, IL- $1 \beta$, and T helper cell responses to Mycobacterium tuberculosis. Front Immunol. 2020 Jul;11:1609.

43 Zhang Q, Zhao K, Shen Q, Han Y, Gu Y, Li X, et al. Tet2 is required to resolve inflammation by recruiting Hdac2 to specifically repress IL6. Nature. 2015 Sep;525(7569):389-93.

44 Lauterbach MA, Hanke JE, Serefidou M, Mangan MSJ, Kolbe C-C, Hess T, et al. Tolllike receptor signaling rewires macrophage metabolism and promotes histone acetylation via ATP-citrate lyase. Immunity. 2019 Dec; 51(6):997-1011.e7.

45 Zhang D, Tang Z, Huang H, Zhou G, Cui C, Weng $\mathrm{Y}$, et al. Metabolic regulation of gene expression by histone lactylation. Nature. 2019 Oct;574(7779):575-80.

46 Chen P, Zuo H, Xiong H, Kolar MJ, Chu Q, Saghatelian A, et al. Gpr132 sensing of lactate mediates tumor-macrophage interplay to promote breast cancer metastasis. Proc Natl Acad Sci U S A. 2017 Jan;114(3):580-5.

47 Meiser J, Krämer L, Sapcariu SC, Battello N, Ghelfi J, D'Herouel AF, et al. Pro-inflammatory macrophages sustain pyruvate oxidation through pyruvate dehydrogenase for the synthesis of itaconate and to enable cytokine expression. J Biol Chem. 2016 Feb;291(8):393246.

48 Zhu X, Long D, Zabalawi M, Ingram B, Yoza $\mathrm{BK}$, Stacpoole PW, et al. Stimulating pyruvate dehydrogenase complex reduces itaconate levels and enhances TCA cycle anabolic bioenergetics in acutely inflamed monocytes. J Leukoc Biol. 2020;107(3):467-84.

49 Liu P-S, Wang H, Li X, Chao T, Teav T, Christen $\mathrm{S}$, et al. $\alpha$-ketoglutarate orchestrates macrophage activation through metabolic and epigenetic reprogramming. Nat Immunol. 2017 Sep;18(9):985-94.
50 Mills EL, Kelly B, Logan A, Costa ASH, Varma $\mathrm{M}$, Bryant CE, et al. Succinate dehydrogenase supports metabolic repurposing of mitochondria to drive inflammatory macrophages. Cell. 2016 Oct;167(2):457-70.e13.

51 Tannahill GM, Curtis AM, Adamik J, Palsson-McDermott EM, McGettrick AF, Goel G, et al. Succinate is an inflammatory signal that induces IL-1 $\beta$ through HIF-1 $\alpha$. Nature. 2013 Apr;496(7444):238-42.

52 Hooftman A, Angiari S, Hester S, Corcoran SE, Runtsch MC, Ling C, et al. The immunomodulatory metabolite itaconate modifies NLRP3 and inhibits inflammasome activation. Cell Metab. 2020 Sep;32(3):468-78.e7.

53 Lampropoulou V, Sergushichev A, Bambouskova M, Nair S, Vincent EE, Loginicheva E, et al. Itaconate links inhibition of succinate dehydrogenase with macrophage metabolic remodeling and regulation of inflammation. Cell Metab. 2016 Jul;24(1):158-66.

54 Mills EL, Ryan DG, Prag HA, Dikovskaya D, Menon D, Zaslona Z, et al. Itaconate is an anti-inflammatory metabolite that activates Nrf2 via alkylation of KEAP1. Nature. 2018 Apr;556(7699):113-7.

55 Liao S-T, Han C, Xu D-Q, Fu X-W, Wang J-S, Kong L-Y. 4-Octyl itaconate inhibits aerobic glycolysis by targeting GAPDH to exert antiinflammatory effects. Nat Commun. 2019 Dec;10(1):5091.

56 Palmieri EM, Gonzalez-Cotto M, Baseler WA, Davies LC, Ghesquière B, Maio N, et al. Nitric oxide orchestrates metabolic rewiring in M1 macrophages by targeting aconitase 2 and pyruvate dehydrogenase. Nat Commun. 2020 Dec;11(1):698.

57 Bailey JD, Diotallevi M, Nicol T, McNeill E, Shaw A, Chuaiphichai $S$, et al. Nitric oxide modulates metabolic remodeling in inflammatory macrophages through TCA cycle regulation and itaconate accumulation. Cell Rep. 2019 Jul;28(1):218-30.e7.

58 Qin W, Qin K, Zhang Y, Jia W, Chen Y, Cheng B, et al. S-glycosylation-based cysteine profiling reveals regulation of glycolysis by itaconate. Nat Chem Biol. 2019 Oct;15(10): 983-91.

59 Blanc M, Hsieh WY, Robertson KA, Watterson S, Shui G, Lacaze P, et al. Host defense against viral infection involves interferon mediated down-regulation of sterol biosynthesis. PLoS Biol. 2011 Mar;9(3):e1000598.

60 Akula MK, Shi M, Jiang Z, Foster CE, Miao D, $\mathrm{Li}$ AS, et al. Control of the innate immune response by the mevalonate pathway. Nat Immunol. 2016 Aug;17(8):922-9.

61 Tan Z, Xie N, Cui H, Moellering DR, Abraham E, Thannickal VJ, et al. Pyruvate dehydrogenase kinase 1 participates in macrophage polarization via regulating glucose metabolism. J Immunol. 2015 Jun; 194(12): 6082-9.
62 Bauerfeld CP, Rastogi R, Pirockinaite G, Lee I, Hüttemann M, Monks B, et al. TLR4-mediated AKT activation is MyD88/TRIF dependent and critical for induction of oxidative phosphorylation and mitochondrial transcription factor A in murine macrophages. J Immunol. 2012 Mar;188(6):2847-57.

63 Lachmandas E, Boutens L, Ratter JM, Hijmans A, Hooiveld GJ, Joosten LAB, et al. Microbial stimulation of different toll-like receptor signalling pathways induces diverse metabolic programmes in human monocytes. Nat Microbiol. 2017 Mar;2(3):16246.

64 Tam VC. Lipidomic profiling of bioactive lipids by mass spectrometry during microbial infections. Semin Immunol. 2013 Oct;25(3):240-8.

65 Wu H, Han Y, Rodriguez Sillke Y, Deng H, Siddiqui S, Treese C, et al. Lipid droplet-dependent fatty acid metabolism controls the immune suppressive phenotype of tumor-associated macrophages. EMBO Mol Med. 2019 Nov;11(11):e10698.

66 Huang SC-C, Everts B, Ivanova Y, O'Sullivan D, Nascimento M, Smith AM, et al. Cell-intrinsic lysosomal lipolysis is essential for alternative activation of macrophages. Nat Immunol. 2014 Sep;15(9):846-55.

67 Wu J-Y, Huang T-W, Hsieh Y-T, Wang Y-F, Yen C-C, Lee G-L, et al. Cancer-derived succinate promotes macrophage polarization and cancer metastasis via succinate receptor. Mol Cell. 2020 Jan;77(2):213-27.e5.

68 Wei X, Song H, Yin L, Rizzo MG, Sidhu R, Covey DF, et al. Fatty acid synthesis configures the plasma membrane for inflammation in diabetes. Nature. 2016 Nov;539(7628):294-8.

69 Malandrino MI, Fucho R, Weber M, Calderon-Dominguez M, Mir JF, Valcarcel L, et al. Enhanced fatty acid oxidation in adipocytes and macrophages reduces lipid-induced triglyceride accumulation and inflammation. Am J Physiol Endocrinol Metab. 2015 May; 308(9):E756-69.

70 Im S-S, Yousef L, Blaschitz C, Liu JZ, Edwards RA, Young SG, et al. Linking lipid metabolism to the innate immune response in macrophages through sterol regulatory element binding protein-1a. Cell Metab. 2011 May; 13(5):540-9.

71 Nomura M, Liu J, Rovira II, Gonzalez-Hurtado E, Lee J, Wolfgang MJ, et al. Fatty acid oxidation in macrophage polarization. Nat Immunol. 2016 Mar;17(3):216-7.

72 Moon J-S, Nakahira K, Chung K-P, DeNicola GM, Koo MJ, Pabón MA, et al. NOX4-dependent fatty acid oxidation promotes NLRP 3 inflammasome activation in macrophages. Nat Med. 2016 Sep;22(9):1002-12.

73 Freigang S, Ampenberger F, Weiss A, Kanneganti T-D, Iwakura Y, Hersberger $\mathrm{M}$, et al. Fatty acid-induced mitochondrial uncoupling elicits inflammasome-independent IL$1 \alpha$ and sterile vascular inflammation in atherosclerosis. Nat Immunol. 2013 Oct;14(10): 1045-53. 
74 Oishi Y, Spann NJ, Link VM, Muse ED, Strid T, Edillor C, et al. SREBP1 contributes to resolution of pro-inflammatory TLR4 signaling by reprogramming fatty acid metabolism. Cell Metab. 2017 Feb;25(2):412-27.

75 Lee J-H, Phelan P, Shin M, Oh B-C, Han X, Im S-S, et al. SREBP-1a-stimulated lipid synthesis is required for macrophage phagocytosis downstream of TLR4-directed mTORC1. Proc Natl Acad Sci U S A. 2018 Dec;115(52): E12228-34.

76 York AG, Williams KJ, Argus JP, Zhou QD, Brar G, Vergnes L, et al. Limiting cholesterol biosynthetic flux spontaneously engages type I IFN signaling. Cell. 2015 Dec;163(7):1716-29.

77 Matalonga J, Glaria E, Bresque M, Escande C, Carbó JM, Kiefer K, et al. The nuclear receptor LXR limits bacterial infection of host macrophages through a mechanism that impacts cellular NAD metabolism. Cell Rep. 2017 Jan; 18(5):1241-55

78 Johansen MD, de Silva K, Plain KM, Whittington RJ, Purdie AC. Mycobacterium avium subspecies paratuberculosis is able to manipulate host lipid metabolism and accumulate cholesterol within macrophages. Microb Pathog. 2019 May;130:44-53.

79 Cortes-Selva D, Elvington AF, Ready A, Rajwa B, Pearce EJ, Randolph GJ, et al. Schistosoma mansoni infection-induced transcriptional changes in hepatic macrophage metabolism correlate with an athero-protective phenotype. Front Immunol. 2018 Nov;9:2580.

80 Huang Y, Morales-Rosado J, Ray J, Myers TG, Kho T, Lu M, et al. Toll-like receptor agonists promote prolonged triglyceride storage in macrophages. J Biol Chem. 2014 Jan;289(5): 3001-12.

81 Ieronymaki E, Daskalaki MG, Lyroni K, Tsatsanis C. Insulin signaling and insulin resistance facilitate trained immunity in macrophages through metabolic and epigenetic changes. Front Immunol. 2019 Jun;10:1330.

82 Ieronymaki E, Theodorakis EM, Lyroni $\mathrm{K}$, Vergadi E, Lagoudaki E, Al-Qahtani A, et al. Insulin resistance in macrophages alters their metabolism and promotes an M2-like phenotype. J Immunol. 2019 Mar;202(6):1786-97.

83 Sok LY, Wollam J, Olefsky JM. An integrated view of immunometabolism. Cell. 2018 Jan; 172(1-2):22-40.

84 Lauterbach MAR, Wunderlich FT. Macrophage function in obesity-induced inflammation and insulin resistance. Pflugers Arch. 2017 Apr;469(3-4):385-96.

85 Costa Rosa LF, Safi DA, Cury Y, Curi R. The effect of insulin on macrophage metabolism and function. Cell Biochem Funct. 1996 Mar; 14(1):33-42.

86 Renier G, Clément I, Desfaits AC, Lambert A. Direct stimulatory effect of insulin-like growth factor-I on monocyte and macrophage tumor necrosis factor-alpha production. Endocrinology. 1996 Nov; 137(11): $4611-8$.
87 Kratz M, Coats BR, Hisert KB, Hagman D, Mutskov V, Peris E, et al. Metabolic dysfunction drives a mechanistically distinct proinflammatory phenotype in adipose tissue macrophages. Cell Metab. 2014 Oct;20(4):614-25.

88 Senokuchi T, Liang C-P, Seimon TA, Han S, Matsumoto M, Banks AS, et al. Forkhead transcription factors (FoxOs) promote apoptosis of insulin-resistant macrophages during cholesterol-induced endoplasmic reticulum stress. Diabetes. 2008 Nov;57(11):2967-76.

89 Mauer J, Chaurasia B, Plum L, Quast T, Hampel B, Blüher M, et al. Myeloid cell-restricted insulin receptor deficiency protects against obesity-induced inflammation and systemic insulin resistance. PLoS Genet. 2010 May; 6(5):e1000938.

90 Spadaro O, Camell CD, Bosurgi L, Nguyen $\mathrm{KY}$, Youm Y-H, Rothlin CV, et al. IGF1 shapes macrophage activation in response to immunometabolic challenge. Cell Rep. 2017 Apr;19(2):225-34.

91 Higashi Y, Sukhanov S, Shai S-Y, Danchuk S, Tang R, Snarski P, et al. Insulin-like growth factor-1 receptor deficiency in macrophages accelerates atherosclerosis and induces an unstable plaque phenotype in apolipoprotein Edeficient mice. Circulation. 2016 Jun;133(23): 2263-78.

92 Knuever J, Willenborg S, Ding X, Akyuz MD, Partridge L, Niessen CM, et al. Myeloid cellrestricted insulin/IGF-1 receptor deficiency protects against skin inflammation. J Immunol. 2015 Dec;195(11):5296-308.

93 Arranz A, Doxaki C, Vergadi E, Martinez de la Torre Y, Vaporidi K, Lagoudaki ED, et al. Akt1 and Akt2 protein kinases differentially contribute to macrophage polarization. Proc Natl Acad Sci U S A. 2012 Jun;109(24):951722.

94 Vergadi E, Vaporidi K, Theodorakis EE, Doxaki C, Lagoudaki E, Ieronymaki E, et al. Akt2 deficiency protects from acute lung injury via alternative macrophage activation and miR-146a induction in mice. J Immunol. 2014 Jan;192(1):394-406.

95 Hedl M, Yan J, Abraham C. IRF5 and IRF5 disease-risk variants increase glycolysis and human M1 macrophage polarization by regulating proximal signaling and Akt2 activation. Cell Rep. 2016 Aug;16(9):2442-55.

96 Babaev VR, Hebron KE, Wiese CB, Toth CL, Ding L, Zhang Y, et al. Macrophage deficiency of Akt2 reduces atherosclerosis in Ldlr null mice. J Lipid Res. 2014 Nov;55(11):2296-308.

97 Jiang H, Westerterp M, Wang C, Zhu Y, Ai D. Macrophage mTORC1 disruption reduces inflammation and insulin resistance in obese mice. Diabetologia. 2014 Nov;57(11):2393404.

98 Zhu L, Yang T, Li L, Sun L, Hou Y, Hu X, et al. TSC1 controls macrophage polarization to prevent inflammatory disease. Nat Commun. 2014 Dec;5(1):4696.
99 Byles V, Covarrubias AJ, Ben-Sahra I, Lamming DW, Sabatini DM, Manning BD, et al. The TSC-mTOR pathway regulates macrophage polarization. Nat Commun. 2013 Dec;4(1):2834.

100 Fang C, Yu J, Luo Y, Chen S, Wang W, Zhao $\mathrm{C}$, et al. Tsc1 is a critical regulator of macrophage survival and function. Cell Physiol Biochem. 2015;36(4):1406-18.

101 Paschoal VA, Belchior T, Amano MT, Burgos-Silva M, Peixoto AS, Magdalon J, et al. Constitutive activation of the nutrient sensor mTORC1 in myeloid cells induced by Tscl deletion protects mice from diet-induced obesity. Mol Nutr Food Res. 2018 Sep; 62(17):e1800283

102 Linke M, Pham HTT, Katholnig K, Schnöller T, Miller A, Demel F, et al. Chronic signaling via the metabolic checkpoint kinase mTORC1 induces macrophage granuloma formation and marks sarcoidosis progression. Nat Immunol. 2017 Mar;18(3):293302.

103 Weichhart T, Haidinger M, Katholnig K, Kopecky C, Poglitsch M, Lassnig C, et al. Inhibition of mTOR blocks the anti-inflammatory effects of glucocorticoids in myeloid immune cells. Blood. 2011 Apr;117(16): 4273-83.

104 Boutens L, Stienstra R. Adipose tissue macrophages: going off track during obesity. $\mathrm{Di}$ abetologia. 2016;59(5):879-94.

105 Ni Y, Ni L, Zhuge F, Xu L, Fu Z, Ota T. Adipose tissue macrophage phenotypes and characteristics: the key to insulin resistance in obesity and metabolic disorders. Obesity. 2020;28(2):225-34.

106 Hill DA, Lim H-W, Kim YH, Ho WY, Foong YH, Nelson VL, et al. Distinct macrophage populations direct inflammatory versus physiological changes in adipose tissue. Proc Natl Acad Sci U S A. 2018;115(22):E5096105.

107 Amano SU, Cohen JL, Vangala P, Tencerova M, Nicoloro SM, Yawe JC, et al. Local proliferation of macrophages contributes to obesity-associated adipose tissue inflammation. Cell Metab. 2014;19(1):162-71.

$108 \mathrm{Li}$ Y, Yun K, Mu R. A review on the biology and properties of adipose tissue macrophages involved in adipose tissue physiological and pathophysiological processes. Lipids Health Dis. 2020;19(1):164.

109 Korf H, Boesch M, Feio-Azevedo R, Smets L, Vandecasteele R, van der Merwe S. Depicting the landscape of adipose tissue-specific macrophages and their immunometabolic signatures during obesity. Immunometabolism. 2020;2(1):e200001

110 Coats BR, Schoenfelt KQ, Barbosa-Lorenzi VC, Peris E, Cui C, Hoffman A, et al. Metabolically activated adipose tissue macrophages perform detrimental and beneficial functions during diet-induced obesity. Cell Rep. 2017 Sep;20(13):3149-61. 
111 Jeong S-J, Lee M-N, Oh GT. The role of macrophage lipophagy in reverse cholesterol transport. Endocrinol Metab. 2017;32(1): 41-6.

112 Dahik VD, Frisdal E, Goff WL. Rewiring of lipid metabolism in adipose tissue macrophages in obesity: impact on insulin resistance and type 2 diabetes. Int J Mol Sci. 2020; 21(15):1-30.

$113 \mathrm{Xu} \mathrm{X,} \mathrm{Grijalva} \mathrm{A,} \mathrm{Skowronski} \mathrm{A,} \mathrm{van} \mathrm{Eijk} \mathrm{M,}$ Serlie MJ, Ferrante AW. Obesity activates a program of lysosomal-dependent lipid metabolism in adipose tissue macrophages independently of classic activation. Cell Metab. 2013 Dec;18(6):816-30.

114 Bechor S, Nachmias D, Elia N, Haim Y, Vatarescu M, Leikin-Frenkel A, et al. Adipose tissue conditioned media support macrophage lipid-droplet biogenesis by interfering with autophagic flux. Biochim Biophys Acta. 2017;1862(9):1001-12.

115 Hadadi-Bechor S, Haim Y, Pecht T, Gat R, Tarnovscki T, Gericke M, et al. Autophagy differentially regulates macrophage lipid handling depending on the lipid substrate (oleic acid vs. acetylated-LDL) and inflammatory activation state. Biochim Biophys Acta Mol Cell Biol Lipids. 2019;1864(12): 158527.

116 Jaitin DA, Adlung L, Thaiss CA, Weiner A, Li B, Descamps H, et al. Lipid-associated macrophages control metabolic homeostasis in a Trem2-dependent manner. Cell. 2019;178(3):686-98.e14.

117 Boutens L, Hooiveld GJ, Dhingra S, Cramer RA, Netea MG, Stienstra R. Unique metabolic activation of adipose tissue macrophages in obesity promotes inflammatory responses. Diabetologia. 2018;61(4):942-53.

118 Serbulea V, Upchurch CM, Ahern KW, Bories G, Voigt P, DeWeese DE, et al. Macrophages sensing oxidized DAMPs reprogram their metabolism to support redox homeostasis and inflammation through a TLR2Syk-ceramide dependent mechanism. Mol Metab. 2018;7:23-34.

119 Serbulea V, Upchurch CM, Schappe MS, Voigt P, DeWeese DE, Desai BN, et al. Macrophage phenotype and bioenergetics are controlled by oxidized phospholipids identified in lean and obese adipose tissue. Proc Natl Acad Sci U S A. 2018 Jul;115(27): E6254-63.

120 Baardman J, Verberk SGS, Prange KHM, van Weeghel M, van der Velden S, Ryan DG, et al. A defective pentose phosphate pathway reduces inflammatory macrophage responses during hypercholesterolemia. Cell Rep. 2018 Nov;25(8):2044-52.e5.

121 Spann NJ, Garmire LX, McDonald JG, Myers DS, Milne SB, Shibata N, et al. Regulated accumulation of desmosterol integrates macrophage lipid metabolism and inflammatory responses. Cell. 2012;151(1):138-52.
122 Willemsen L, Neele AE, van der Velden S, Prange KHM, den Toom M, van Roomen CPAA, et al. Peritoneal macrophages have an impaired immune response in obesity which can be reversed by subsequent weight loss. BMJ Open Diabetes Res Care. 2019; 7(1):e000751.

123 Shao X, Xiang S, Chen Y, Zhang N, Cao J, $\mathrm{Zhu} \mathrm{H}$, et al. Inhibition of M2-like macrophages by all-trans retinoic acid prevents cancer initiation and stemness in osteosarcoma cells. Acta Pharmacol Sin. 2019 Oct; 40(10):1343-50.

124 Zeng X-Y, Xie H, Yuan J, Jiang X-Y, Yong $\mathrm{J}-\mathrm{H}$, Zeng D, et al. M2-like tumor-associated macrophages-secreted EGF promotes epithelial ovarian cancer metastasis via activating EGFR-ERK signaling and suppressing lncRNA LIMT expression. Cancer Biol Ther. 2019 Jul;20(7):956-66.

125 Liang ZX, Liu HS, Wang FW, Xiong L, Zhou $\mathrm{C}, \mathrm{Hu} \mathrm{T}$, et al. LncRNA RPPH1 promotes colorectal cancer metastasis by interacting with TUBB3 and by promoting exosomesmediated macrophage M2 polarization. Cell Death Dis. 2019 Nov;10(11):829.

126 Yamaguchi T, Fushida S, Yamamoto Y, Tsukada T, Kinoshita J, Oyama K, et al. Tumorassociated macrophages of the M2 phenotype contribute to progression in gastric cancer with peritoneal dissemination. Gastric Cancer. 2016 Oct;19(4):1052-65.

127 Su P, Wang Q, Bi E, Ma X, Liu L, Yang M, et al. Enhanced lipid accumulation and metabolism are required for the differentiation and activation of tumor-associated macrophages. Cancer Res. 2020 Apr;80(7):143850 .

128 Wu L, Zhang X, Zheng L, Zhao H, Yan G, Zhang Q, et al. RIPK3 Orchestrates fatty acid metabolism in tumor-associated macrophages and hepatocarcinogenesis. Cancer Immunol Res. 2020 May;8(5):710-21.

129 Niu Z, Shi Q, Zhang W, Shu Y, Yang N, Chen $B$, et al. Caspase-1 cleaves PPAR $\gamma$ for potentiating the pro-tumor action of TAMs. Nat Commun. 2017 Dec;8(1):766.

130 Xiang W, Shi R, Kang X, Zhang X, Chen P, Zhang L, et al. Monoacylglycerol lipase regulates cannabinoid receptor 2-dependent macrophage activation and cancer progression. Nat Commun. 2018 Dec;9(1):2574.

131 Huang SC-C, Smith AM, Everts B, Colonna M, Pearce EL, Schilling JD, et al. Metabolic reprogramming mediated by the $\mathrm{mTORC} 2$ IRF4 signaling axis is essential for macrophage alternative activation. Immunity. 2016 Oct;45(4):817-30.

132 Liu D, Chang C, Lu N, Wang X, Lu Q, Ren $\mathrm{X}$, et al. Comprehensive proteomics analysis reveals metabolic reprogramming of tumorassociated macrophages stimulated by the tumor microenvironment. J Proteome Res. 2017 Jan;16(1):288-97.
133 Penny HL, Sieow JL, Adriani G, Yeap WH See Chi Ee P, San Luis B, et al. Warburg metabolism in tumor-conditioned macrophages promotes metastasis in human pancreatic ductal adenocarcinoma. Oncoimmunology. 2016 Aug;5(8):e1191731.

134 Zhihua Y, Yulin T, Yibo W, Wei D, Yin C, Jiahao $\mathrm{X}$, et al. Hypoxia decreases macrophage glycolysis and M1 percentage by targeting microRNA-30c and mTOR in human gastric cancer. Cancer Sci. 2019 Aug;110(8): 2368-77.

135 Orillion A, Damayanti NP, Shen L, Adelaiye-Ogala R, Affronti H, Elbanna M, et al. Dietary protein restriction reprograms tumorassociated macrophages and enhances immunotherapy. Clin Cancer Res. 2018 Dec; 24(24):6383-95.

136 Altman BJ, Stine ZE, Dang CV. From Krebs to clinic: glutamine metabolism to cancer therapy. Nat Rev Cancer. 2016 Oct;16(10): 619-34.

137 Oh M-H, Sun I-H, Zhao L, Leone RD, Sun $\mathrm{I}-\mathrm{M}, \mathrm{Xu} \mathrm{W}$, et al. Targeting glutamine metabolism enhances tumor-specific immunity by modulating suppressive myeloid cells. $]$ Clin Invest. 2020 Jun;130(7):3865-84.

138 Palmieri EM, Menga A, Martín-Pérez R, Quinto A, Riera-Domingo C, De Tullio G, et al. Pharmacologic or genetic targeting of glutamine synthetase skews macrophages toward an M1-like phenotype and inhibits tumor metastasis. Cell Rep. 2017 Aug;20(7):1654-66.

139 Sosnowska A, Czystowska-Kuzmicz M, Golab J. Extracellular vesicles released by ovarian carcinoma contain arginase 1 that mitigates antitumor immune response. Oncoimmunology. 2019 Nov;8(11):e1655370.

140 Sharda DR, Yu S, Ray M, Squadrito ML, De Palma M, Wynn TA, et al. Regulation of macrophage arginase expression and tumor growth by the Ron receptor tyrosine kinase. J Immunol. 2011;187(5):2181-92.

141 Devalaraja S, To TKJ, Folkert IW, Natesan R, Alam MZ, Li M, et al. Tumor-derived retinoic acid regulates intratumoral monocyte differentiation to promote immune suppression. Cell. 2020 Mar;180(6):1098-114.e16.

142 Silva LS, Poschet G, Nonnenmacher Y, Becker HM, Sapcariu S, Gaupel A, et al. Branched-chain ketoacids secreted by glioblastoma cells via MCT1 modulate macrophage phenotype. EMBO Rep. 2017 Dec; 18(12):2172-85.

143 Hattori A, Tsunoda M, Konuma T, Kobayashi M, Nagy T, Glushka J, et al. Cancer progression by reprogrammed BCAA metabolism in myeloid leukaemia. Nature. 2017 May;545(7655):500-4.

144 Carmona-Fontaine C, Bucci V, Akkari L, Deforet M, Joyce JA, Xavier JB. Emergence of spatial structure in the tumor microenvironment due to the Warburg effect. Proc Natl Acad Sci U S A. 2013 Nov;110(48): 19402-7. 
145 Zhang L, Li S. Lactic acid promotes macrophage polarization through MCT-HIFla signaling in gastric cancer. Exp Cell Res. 2020 Mar;388(2):111846.

146 Morris JP, Yashinskie JJ, Koche R, Chandwani R, Tian S, Chen C-C, et al. a-Ketoglutarate links p53 to cell fate during tumour suppression. Nature. 2019 Sep; 573(7775):595-9.

147 Liu PS, Wang H, Li X, Chao T, Teav T, Christen S, et al. a-ketoglutarate orchestrates macrophage activation through metabolic and epigenetic reprogramming. Nat Immunol. 2017;18(9):985-94.

148 Weiss JM, Davies LC, Karwan M, Ileva L, Ozaki MK, Cheng RYS, et al. Itaconic acid mediates crosstalk between macrophage metabolism and peritoneal tumors. J Clin Invest. 2018 Aug;128(9):3794-805.

149 Hoefflin R, Harlander S, Schäfer S, Metzger $\mathrm{P}$, Kuo F, Schönenberger D, et al. HIF-1a and HIF-2a differently regulate tumour development and inflammation of clear cell renal cell carcinoma in mice. Nat Commun. 2020 Dec;11(1):4111.

150 Saha J, Sarkar D, Pramanik A, Mahanti K, Adhikary A, Bhattacharyya S. PGE2-HIF1a reciprocal induction regulates migration, phenotypic alteration and immunosuppressive capacity of macrophages in tumor microenvironment. Life Sci. 2020 Jul;253:117731.
151 Bao D, Zhao J, Zhou X, Yang Q, Chen Y, Zhu J, et al. Mitochondrial fission-induced $\mathrm{mtD}$ NA stress promotes tumor-associated macrophage infiltration and HCC progression. Oncogene. 2019 Jun;38(25):5007-20.

152 Yang J-B, Zhao Z-B, Liu Q-Z, Hu T-D, Long $\mathrm{J}$, Yan $\mathrm{K}$, et al. FoxO1 is a regulator of MHCII expression and anti-tumor effect of tumor-associated macrophages. Oncogene. 2018 Mar;37(9):1192-204.

153 Wang W, Liu Y, Guo J, He H, Mi X, Chen C, et al. miR-100 maintains phenotype of tumor-associated macrophages by targeting mTOR to promote tumor metastasis via Stat5a/IL-1ra pathway in mouse breast cancer. Oncogenesis. 2018 Dec;7(12):97.

154 Hou P, Luo L, Chen H, Chen Q, Bian X, Wu $\mathrm{S}$, et al. Ectosomal PKM2 promotes HCC by inducing macrophage differentiation and remodeling the tumor microenvironment. Mol Cell. 2020 Jun;78(6):1192-206.e10.

155 Cooks T, Pateras IS, Jenkins LM, Patel KM, Robles AI, Morris J, et al. Mutant p53 cancers reprogram macrophages to tumor supporting macrophages via exosomal miR1246. Nat Commun. 2018 Dec;9(1):771.
156 Gerloff D, Lützkendorf J, Moritz RKC, Wersig T, Mäder K, Müller LP, et al. Melanomaderived exosomal miR-125b-5p educates tumor associated macrophages (TAMs) by targeting lysosomal acid lipase A (LIPA) Cancers. 2020 Feb;12(2):464.

157 Xiao M, Zhang J, Chen W, Chen W. M1-like tumor-associated macrophages activated by exosome-transferred THBS1 promote malignant migration in oral squamous cell carcinoma. J Exp Clin Cancer Res. 2018 Dec; 37(1):143.

158 Roux C, Jafari SM, Shinde R, Duncan G, Cescon DW, Silvester J, et al. Reactive oxygen species modulate macrophage immunosuppressive phenotype through the up-regulation of PD-L1. Proc Natl Acad Sci U S A. 2019 Mar;116(10):4326-35.

159 Griess B, Mir S, Datta K, Teoh-Fitzgerald M. Scavenging reactive oxygen species selectively inhibits M2 macrophage polarization and their pro-tumorigenic function in part, via Stat3 suppression. Free Radic Biol Med. 2020 Feb;147:48-60.

160 Alexander RK, Liou Y-H, Knudsen NH, Starost $\mathrm{KA}, \mathrm{Xu} \mathrm{C}$, Hyde $\mathrm{AL}$, et al. Bmal1 integrates mitochondrial metabolism and macrophage activation. Elife. 2020 May;9: e54090. 